\title{
SHORT-TERM VARIABILITY OF SAGITTARIUS A* AT MILLIMETER WAVELENGTHS
}

\author{
T. TSUTSUMI \\ Nobeyama Radio Observatory, NAOJ \\ Minamimaki, Minamisaku, Nagano 384-13, Japan \\ T. KAWABATA \\ Bisei Astronomical Observatory \\ Okayama, Japan
}

AND

A. MIYAZAKI AND M. TSUBOI

Institute of Astrophysics and Planetary Science, Ibaraki University, Ibaraki, Japan

Time variability of $\mathrm{Sgr} \mathrm{A}^{*}$ at $\mathrm{cm}$ wavelengths is well known phenomenon and has been studied by several authors (e.g. Lo 1989; Zhao et al. 1992). On the other hand, variability at millimeter wavelengths is not well established. Wright \& Backer (1993) reported significant flux variations at $\lambda=3.4 \mathrm{~mm}$ in a month during the decay of a flare observed by the Very Large Array in 1990. However others found no variability at millimeter and submillimeter wavelengths (Gwinn et al. 1991; Zylka et al. 1995). Emission from extended component surrounding $\mathrm{Sgr} \mathrm{A}^{*}$ is significant even at millimeter wavelengths (e.g. Tsuboi et al. 1988), thus it is important to observe with a narrower beam to isolate the compact component for accurate measurement of variability of Sgr A*. Here we present some results from an on-going millimeter monitoring program of $\mathrm{Sgr} \mathrm{A}^{*}$ with the Nobeyama Millimeter Array (NMA).

Between the two observing seasons (from February 1996 to April 1997), we obtained total of 8 epochs at $101.7 \mathrm{GHz}$ and 5 epochs at $146.5 \mathrm{GHz}$ of the NMA data. A typical size of synthesized beam is $4.0^{\prime \prime} \times 2.0^{\prime \prime}(\mathrm{FWHM})$ at $102 \mathrm{GHz}$. Uncertainties in flux calibration are estimated to be $\sim 15 \%$ for $102 \mathrm{GHz}$ and $\sim 20 \%$ for $147 \mathrm{GHz}$. The flux densities measured on the maps are reduced due to decorrelation by phase fluctuation. The flux densities previously presented (Tsutsumi et al. 1997) are corrected for this effect 


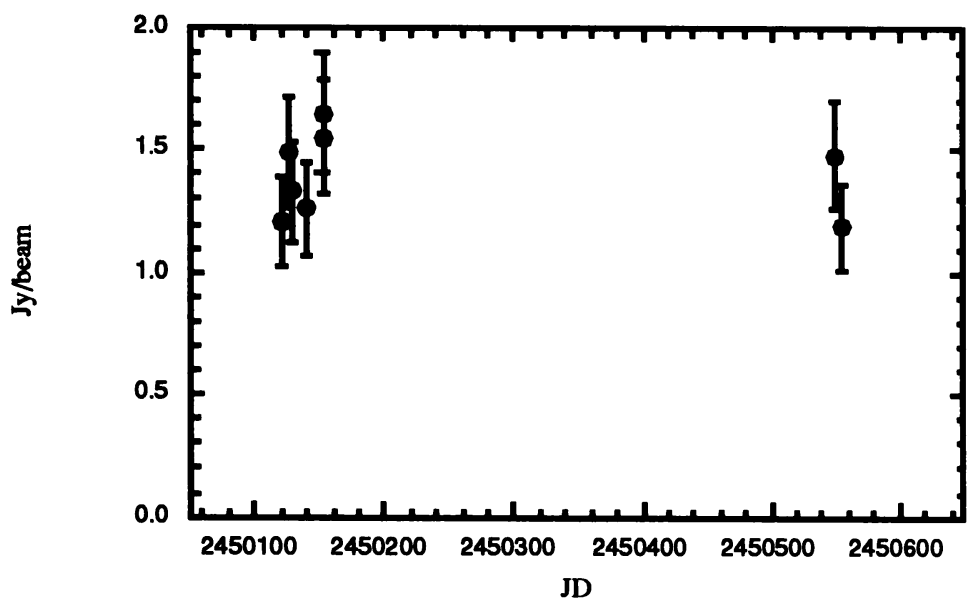

Figure 1. Time variability of Sgr $\mathrm{A}^{*}$ at $102 \mathrm{GHz}$ during Feb. 1996 to Apr. 1887

and shown here in Figure 1. Although the uncertainties in the absolute flux densities are large, the variability of $\mathrm{Sgr} \mathrm{A}^{*}$ at $102 \mathrm{GHz}$ of $\leq 30 \%$ in a few weeks is suggested in this data.

Spectral indices are calculated from the $102 \mathrm{GHz}$ and $147 \mathrm{GHz}$ data for 1996 and from two sideband data at $100 \mathrm{GHz}(89.7 \mathrm{GHz}$ and $101.7 \mathrm{GHz})$ for 1997 that are separately imaged. Spectral indices as determined from the simultaneous observations at the two $100 \mathrm{GHz}$ sidebands are $\alpha \sim 0.3$ for both epochs in 1997. These are, within the uncertainties, consitent with the model for quasi-monoenergetic synchrotron emitting electrons (Beckert et al. 1996) that predict the observed spectrum as $S \propto \nu^{+0.33}$. For the 1996 data, spectral indices between 100 and $150 \mathrm{GHz}$ are $\alpha=+1.0 \pm 0.9$ on 8 Feb. 1996 and $\alpha=-1.8 \pm 0.9$ on 11 Mar. 1996, indicating possible variations in the spectral index. Since large uncertainties are associated with these values it needs to be confirmed by further monitoring at these wavelengths.

\section{References}

Beckert, T., Duschl, W. J., Mezger, P.G., and Zylka, R. 1996, A\&A 307, 450.

Gwinn, C. R., et al. 1991, Ap. J. 381, L43.

Lo, K.Y. 1989 in The Center of the Galaxy, M. Morris ed., 527.

Tsuboi, M., et al. 1988, PASJ 40, 665.

Tsutsumi, T., Kawabata, T., Miyazaki, A., and Tsuboi, M. 1997, in IAU Colloq.164 Radio

Emission from Galactic and Extragalactic Compact Sources, Zensus, J. A., Wrobel, J.M., and Taylor, G. B. eds., in press.

Wright, M. C. H. and Backer, D. C. 1993, Ap. J 417, 560.

Zhao, J.-H., Goss, W. M., Lo, K.-Y., and Eker, R. D. 1992 in Relationships between active galactic nuclei and starburst galaxies, A. Filippenko ed., 295.

Zylka, R., et al. 1995, A\&A, 297, 83. 